\title{
A daunting journey: A qualitative comparative study of women's experiences of accessing midwifery care
}

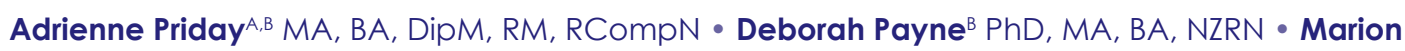
Hunter $^{B}$ DHSC, MA, BA, ADN, RM, RGON

${ }^{\mathrm{A}}$ Corresponding Author: adrienne. priday@aut.co.nz

${ }^{B}$ School of

Midwifery, Auckland University of

Technology, Aotearoa New Zealand

\begin{abstract}
Background: Early engagement with a maternity carer is recommended as a means of reducing stillbirth and neonatal mortality. This is especially important for women who live in high deprivation areas, as these areas have been associated with late access to maternity/midwifery care and significantly higher rates of stillbirth and neonatal mortality. Co-locating midwives at general practitioner (GP) clinics in such an area was established with the aim of facilitating women's early access to midwifery care.
\end{abstract}

Aim: To explore the experience of multiparous women who live in socio-economically deprived communities within the Counties Manukau Health region and who accessed the services of midwives at co-located clinics.

Method: Interpretive descriptive methodology was used to explore the experiences of each woman before and after using a co-located midwifery clinic. One-to-one, semi-structured interviews were undertaken and data examined using thematic analysis.

Findings: The eight women interviewed found accessing Lead Maternity Care (LMC) midwives during early pregnancy a daunting journey before being able to use a co-located clinic in the Counties Manukau Health region. Barriers identified were: a lack of knowledge about how to find a LMC midwife, limited finance and limited time. These impacted on women's ability and confidence to find a suitable LMC midwife. The women expressed the need for help to circumvent the maternity care maze through receiving a recommendation for a LMC midwife and having access to a midwife co-located at their GP clinic.

Conclusions: The participants encountered numerous barriers accessing early LMC midwifery care. Enablers to accessing early LMC midwifery care include receiving recommendations from GP clinic personnel, and midwives being co-located at GP clinics to make maternity care convenient and with a smooth transition from GP to LMC midwife care.

Keywords: accessing antenatal care, co-located midwifery clinics, high deprivation, interpretive description, midwifery care

\section{INTRODUCTION}

Aotearoa New Zealand's (Aotearoa NZ's) maternity services are unique as they are based on women-centred community care, integrating with secondary/tertiary hospital services when required. Women select a lead maternity carer (LMC) - who can be a midwife, a general practitioner (GP) or an obstetrician - to provide and coordinate care during their childbirth experience. In $2018,94.5 \%$ of women who registered with a LMC chose a midwife (Ministry of Health [MOH], 2020). LMC midwives work autonomously and collaboratively within the midwifery scope of practice (Midwifery Council of New Zealand, 2010).

Women living in areas of high socio-economic deprivation are less likely to register early with a midwife and are at increased risk of experiencing stillbirth or neonatal death (Perinatal \& Maternal Mortality Review Committee [PMMRC], 2018).
The Counties Manukau Health (CMH) region includes a large number of families/whānau living with high social deprivation. Women living in this region have been identified as having higher perinatal mortality rates than women in areas of higher socio-economic status across Aotearoa NZ (Atkinson et al., 2014; CMH, 2014; Jackson, 2011; PMMRC, 2018). Data from the Aotearoa NZ PMMRC demonstrate better maternity outcomes for women and babies when antenatal care is commenced early in the pregnancy (PMMRC, 2018); preferably before 10 weeks' gestation $(\mathrm{MOH}, 2018$; National Institute for Health \& Clinical Excellence, 2010). The Aotearoa NZ National Maternity Monitoring Group similarly prioritises timely registration with a LMC (MOH, 2018).

Following a 2011 MOH-commissioned report, Priday and McAra-Couper (2016) captured statistical outcomes achieved 
over 15 years by the model of midwifery clinics co-located at GP clinics in the $\mathrm{CMH}$ region.

In 2012, the $\mathrm{MOH}$ presented its report to $\mathrm{CMH}$. This report then informed the External Review of Maternity Care in the Counties Manukau District (Paterson et al., 2012). The report concluded that women utilising co-located midwifery clinics had a higher rate of both early registration with antenatal care and positive birth outcomes. Thus, the external review recommended the co-located maternity service model be replicated and expanded throughout other high deprivation communities in $\mathrm{CMH}$. From 2013 to 2015 , CMH implemented the recommendations with the aim of women engaging early within the support of co-located midwifery clinics in these communities.

To date there has been no research capturing the women's experiences of utilising this expansion of co-located midwifery clinics at GP clinics. The aims of this interpretive descriptive study were: 1) to explore women's experiences of accessing and engaging with midwifery care before and after using a co-located midwifery clinic; 2) to determine if the co-located clinics serve women's needs; and 3) to offer recommendations, if warranted, for improved services based on findings.

\section{METHOD}

Interpretive description was used as the methodology as it was fit for purpose to address the research question, which asked: What are the experiences of multiparous women who live in socioeconomically deprived communities of the $\mathrm{CMH}$ region who have received midwifery care located at their GP clinic? Thorne's (2008) concept of interpretive description is an inductive approach designed to explore clinically relevant phenomena, particularly in social and primary health care environments. Interpretive description is particularly applicable for understanding complex experiential clinical and practical phenomena, events or processes (Berterö, 2015; Thorne, 2008), such as this study where women interface with a primary health service specifically designed for pregnant women's health care. This research approach encouraged women to speak freely, eliciting rich data.

\section{Ethics, confidentiality and consent}

Ethical approval was granted by the Auckland University of Technology Ethics Committee in June 2016 (ref: 16/320). As we anticipated most participants would be of Māori or Pasifika ethnicity, consultation occurred throughout the study with two representative midwives, one from Nga Maia and the other from Pasifika Midwives Aotearoa. Confidentiality was maintained by using pseudonyms and removing any identifying information given in the interviews. All interviews were transcribed verbatim by a transcriptionist who signed a confidentiality agreement. Written consent from participants was obtained prior to commencing and digitally recording the interviews.

\section{Participant recruitment}

GP clinic personnel and midwives who worked in co-located clinics in the $\mathrm{CMH}$ region were approached to give information sheets to eligible women. Women interested in the study were invited to phone or text their interest. The inclusion criteria were multiparous women who: a) in the last five years had received previous pregnancy care at a location other than their GP clinic, and with subsequent pregnancies had received midwifery care from midwives located at their GP clinic; (b) were conversant in English; (c) were over 18 years of age; and (d) were residing in the high socio-economic deprivation areas of $\mathrm{CMH}$. The exclusion criterion was women who had received past midwifery care from the first author or her back-up midwife. In total, eight women were recruited and interviewed over a three-month period (Table 1).

\begin{tabular}{ll} 
Table 1. Participant demographics for current or most \\
recent pregnancy $(\mathbf{n = 8})$ \\
Ethnicity \\
Māori \\
Pasifika & 2 \\
Pasifika/Māori & 2 \\
Māori/European & 1 \\
Indian & 1 \\
European & 1 \\
Age range & 1 \\
Gravida & $28-38$ years \\
Gravida 2 & \\
Gravida 4 & 5 \\
Gravida 5 & 1 \\
Gravida 6 & 1 \\
Distance from home to GP clinic & 1 \\
1-10 mins drive & 3 \\
10 mins walk & \\
Unable to drive & 3 \\
\hline
\end{tabular}

Data were collected through individual, semi-structured, faceto-face interviews at a location of the woman's choosing. The interviews were participant led and guided by an interview prompt sheet to keep the dialogue related to the research topic. Interviews began by asking demographic questions which served as springboards; women often recalled further information when responding to the demographic questions about accessing midwifery care. For example, "Where do you live in relation to your GP clinic and where have you accessed a midwife?" was then further explored by asking "How long does it take you to walk or drive to these clinics?" These questions were reviewed after each interview to fine-tune the exploration of the topic with the evolution of the interviews. For example, in the first interview the participant described in-depth being able to walk to her clinic when driving or vehicle transport was not possible, hence in her case the initial question was expanded upon; for example, "Tell me about driving in a car when you are pregnant?"

As we wanted to interview women who had experienced both co-located and non-co-located midwifery care, we explored the processes each woman undertook to find a midwife. The interviews lasted between 40 and 70 minutes and were audio recorded; handwritten notes taken during the interview aided management of the record-keeping processes.

In keeping with descriptive interpretive methodology, interviews ceased once the data from the final participants were seen as repeating similar themes.

\section{Data analysis}

The data were analysed using thematic analysis: the process of discovering recognisable patterns or themes captured within the participants' recollections about the topic in question (Thorne, 2008). By using an iterative and inductive method of analysis guided by Thorne, Kirkham and O'Flynn-Magee (2004), Thorne, Con et al. (2004) and Thorne (2008), we were able to generate rich data from the women's experiences. The analysis process was constantly open to discussion, which allowed the data to be coded according to similarities; from these codes, themes were 
developed. The complexities of health and social issues during these care episodes were also looked for and explained.

The trustworthiness criteria - credibility, dependability, confirmability and transferability for judging the data analysis process - were informed by Guba and Lincoln (1982). To meet these, we employed multiple strategies throughout the research process. They included: purposive sampling of participants who had experiences of accessing midwifery care both independently and via co-location with their GP practice; gaining participants' perspectives and describing these accurately; prolonged engagement with the data; keeping a decision trail and practising reflexivity; and presenting preliminary findings to a group of peer midwives. Participants were each sent a copy of their transcript for any changes or comments.

To further promote trustworthiness, the first author discussed her experiences of working in and researching co-located clinics, revealing any assumptions and prejudices (Smythe, 2012; Temple, 1997). This was especially important given the 'insider-outsider' phenomenon (Dwyer \& Buckle, 2009) which can result in bias within qualitative research, where the researcher is inseparable from the study. During the process of data analysis, presumptions were challenged to ensure codes and themes arose solely from the participants' data.

\section{FINDINGS}

Two themes emerged from participants' experiences accessing colocated midwifery care at their GP clinic: "a daunting journey" and "circumventing the maternity care maze". All the participants described their previous journey of accessing midwifery care as daunting, whereas being able to access a co-located midwife circumvented the maternity care maze.

\section{A daunting journey: Accessing midwifery care}

All participants found the process of accessing LMC midwifery care onerous. Most women signalled knowing how to find a LMC midwife as the first barrier to accessing early midwifery care:

There should be an information sheet or something for people who don't know what the next step is... there needs to be more information on what the next step is and what we need to do to find a midwife. How do I do that? Who do I turn to? Who can you suggest...It is a daunting task finding the right person. (Sui)

Sui explained her concerns; the process started with having her pregnancy confirmed by her GP. However, she then found the next step an intimidating and discouraging struggle. The paucity of information regarding how to connect with a midwife was a major obstacle for Sui.

Phoning to ask if a specific midwife could care for them could frustrate participants' efforts to access early midwifery care:

\section{I rung my old midwife and asked if she had any recommendations and she said to go on a website... it had so many midwives. It was a long process. Quite daunting having to go through all these ladies and you'd find one and she'd be booked out. To be honest... I gave up, it was just too daunting. (Mohi)}

The established system for finding a midwife was assumed to be helpful by Mohi's previous midwife. However, for Mohi the process of accessing the website, identifying possible midwives, contacting them and then finding they were already fully booked, meant she did not persevere with phoning further midwives. The website, although accessible, was not in her case a helpful resource.
Nisha also grappled with the pathways to finding a midwife:

There's a lot of midwives' numbers and names, which one to ring? ...I have to decide. How am I supposed to know they're good or bad? I didn't think it was a good idea ringing all of them up because I don't know where or who they are. (Nisha)

Nisha found having to phone many midwives challenging, let alone knowing what questions to ask to ascertain if they were going to satisfy her quest for a "good" midwife. Nisha wanted assurance that her choice of midwife would bring safe care for her and her baby; she was unable to see how it would happen with this system of finding a midwife.

Another barrier was the level of the woman's confidence. Most participants were uncomfortable and fearful of being "bold" with midwives they did not know. They did not wish to ask the midwives to qualify themselves and feared being rejected by a midwife. Phoning and asking for the service of a stranger required courage:

People sometimes don't have the confidence to ring up and ask. (Mia)

\section{I don't want to ring them up and ask them how much experience they have. (Nisha)}

Not being able to drive was another barrier to engaging a midwife. Some participants cited reasons, like cultural beliefs, for not wishing to drive and travel distances.

In our tradition we're not allowed to drive in the first
stage of pregnancy because we believe that if the road is
pondit [pot holed] it will make the baby inside easy to get
a miscarriage... I think I should find a new one [midwife]
that is closer to home. I can't manage to drive there, it's
long, not just around the corner where the [GP] clinic is
[which is] a five-minute walk [from Ana's home]. If I can
walk it would be good if I don't want to drive. (Ana)

For her first pregnancy Ana had a co-located midwife who was recommended for her. Ana wished to reconnect with the same midwife but the midwife's new clinic was about a 20-minute drive from Ana's home. The midwife did try to accommodate Ana's reluctance to drive to this new location by visiting Ana at her home. However, when home visits were not possible, Ana drove herself to the new clinic. Ana's family held the cultural belief around being in a car that, in the early phase of pregnancy, this might cause a miscarriage. Secondly, there was additional distance to travel to see her doctor if this was recommended by the midwife. These challenges culminated in Ana considering finding midwifery care closer to her home.

The final barriers participants discussed were limited finances and time, citing limited phone credit, lack of or limited internet access, parking costs and being time poor.

I don't always have credit on phone and [if it] goes to message, credit is wasted...I don't use buses, too much money and I don't know where they go. (Ana)

People sometimes don't even have access to the internet... In the city you pay for parking. At a hospital you pay for parking. At the [GP] clinic you don't... it's easy and great! Ahh the to and fro, like the travel distance plus parking. My chemist is right downstairs so everything was all in one. If you can get your doctors, your midwife, your lab tests, your chemist, all in the one facility, it's like a cost bonus! (Mia) 
Ana's and Mia's comments challenge the assumption that everyone can afford these costs or has easy access to a phone and the internet. They also highlight the time that antenatal care can take, especially when services are not within an easy distance to one another. All these factors required consideration and were hurdles to the attending of midwifery appointments.

\section{Circumventing the maternity care maze}

Participants wanted help circumventing the maternity care maze. When reflecting on their experiences of co-located midwifery care, all valued receiving a LMC midwife recommendation from the GP clinic personnel. It relieved the burden of finding a midwife through their own efforts.

Rata describes receiving guidance:

Our [GP] nurse, she always looks out for me and my son, so the moment she said this person [the midwife] was good, it was a relief. She could have said I could go to Arnold Schwarzenegger and I still would have believed her. It was awesome. (Rata)

It was about needing reassurance that the midwife could do her job and do it well. Having her GP's practice nurse recommend a midwife relieved her of this concern. Similarly, other women appreciated receiving a recommendation from their trusted health practitioner:

\section{Having my GP rate her [the midwife] quite highly made the decision so much easier...I value her [the GP's] opinion. (Kelly)}

Kelly had a relationship of trust with her GP; the midwife recommendation was highly regarded and facilitated her finding a suitable midwife.

All participants valued having a midwife co-located at their GP clinic. It made maternity care a smooth transition from GP to LMC midwife. It also enabled women to address other family/ whānau health needs during the same visit:

It was convenient and stress free, when you're working you have kids, other commitments... It takes a bit of work off your plate having a midwife out of your GP clinic, convenient. (Kelly)

It was great, easy getting the vaccinations [whooping cough and flu]. I'm so busy with the kids and you know it makes it a whole lot easier... I went through a bit of depression, so my midwife helped me through that, to get on to some sort of counselling and work together with my doctor. (Mia)

To not drive elsewhere. Just to have her [the midwife] in the same location with the practice...Yeah, like with the pharmacy and everything on the same location. Not having to go out of my way to see a midwife. (Alia)

As for most of the participants, Kelly, Mia and Alia were busy women, having multiple demands such as work and extended family/whānau responsibilities. Having midwifery care, their GP and other health services located together saved time and money. Co-location eased both the process of enlisting a midwife and also facilitated the opportunity for the GP and midwife to work together. Furthermore, for these busy women, being able to piggyback other health-related appointments on to an antenatal visit was a convenient timesaver.

Being familiar with the location and the people at their GP clinic assisted them to engage with midwifery care, as participants felt at ease. For example:
It [midwifery care] was in a space which I was familiar with. I know where it is, it's really close to home. It feels more comfortable walking in there, it's intrinsically important for me. Being part-Mãori, I can't stress enough how much better it feels going somewhere where you know, my mum trusts, my dad did, my husband does, my son goes too. You know, they know my whole history. (Rata)

Rata noted the importance of her family connectedness with the GP clinic. She described herself as feeling more relaxed receiving midwifery care for her second pregnancy in a known, trusted environment which her family/whānau frequent and where they too trust the GP clinic. Additionally, being in a place where her health history is known was seen as another positive factor.

Lastly, participants valued the co-located midwife and GP being able to easily communicate between each other, in person, about their health, pregnancy outcomes and family planning needs:

When I did [my baby's] 6 weeks vaccination my midwife told everything to my doctor, she actually went face to face [with the GP] about family planning, what I have to do, which one she suggested is better for me. Also, she [the baby] had a cord problem, so my doctor also knows because my midwife went there. And the midwife called me and she goes "I spoke to your GP and that's what she suggested", all these things. So, it was easy. I don't have to go to my GP and say everything. So, she did the half of the job for me after postnatal. Yeah, and also my GP goes "oh I heard about you, [your midwife] told me all about you, your labour was very good". (Nisha)

When Nisha attended her first GP appointment after the midwife discharged her, she valued the communication that had occurred between the midwife and her GP. It took the pressure off Nisha having to explain to the GP aspects of her maternity care such as family planning and the concern re baby's cord.

\section{DISCUSSION}

The findings in this study identified that our participants encountered their first barrier at the entry point of the LMC midwifery system - knowing how to find a midwife. All the women experienced difficulties having to find their own midwife with their first pregnancy and described this as a daunting journey. In comparison, having a midwife co-located at the GP clinic, or a LMC midwife being recommended to them by their GP or practice nurse, circumvented the time, cost and stress that finding a midwife entailed. Women in this study identified being appreciative of receiving a recommendation, guidance and information from their GP or practice nurse to help them take the steps needed between pregnancy confirmation to finding a midwife. Lovell (1996) asserted that providing pregnant women with information and support will empower them to have choices and make decisions within their parenting journey.

It is important to note that all the women in this study went to their GP to have their pregnancy confirmed. In Aotearoa NZ a large proportion of women first see a GP to confirm their pregnancy (Corbett et al., 2013; Jackson, 2011). It is therefore important that GPs and/or practice nurses, during their conversations with women at the time of pregnancy confirmation, support navigation to local LMC midwives. Aotearoa NZ and overseas literature supports improving public health communication and interventions to increase specific knowledge on the importance of accessing early antenatal care (Aquino et al., 2015; Copland et al., 2011; Griffiths et al., 2013; Lindquist et al., 2014; Makowharemahihi et al., 2014; $\mathrm{MOH}, 2017)$. 
The women in this study described how they found the process of phoning LMC midwives to check availability an exhausting process. The midwife contact list, given to women by the GP clinic personnel, had limited information, providing only the midwife's phone number and geographical location. Even more perplexing, once having found an available midwife, the women were then required to converse with the midwife about their needs. The women felt uncomfortable with this independent way of finding a midwife; and this was an obstacle for those women who preferred the GP or practice nurse's help to recommend a midwife. The GP or practice nurse were viewed as trusted advisors who provided guidance as to which midwife would meet the woman's needs.

For Māori and Pasifika women, having to ask a health professional to care for them is a culturally and socially uncomfortable task and avoided where possible (Bartholomew et al., 2015; Corbett et al., 2013; Makowharemahihi et al., 2014). On the other hand, for the women in this study, to have their GP or practice nurse recommend a LMC midwife saved them the embarrassment of either having to directly contact a midwife, or not making contact at all, thus delaying care. Our findings echo/reflect those of Makowharemahihi et al. (2014) who found that women in their study phoned many different midwives only to find them unavailable, or their messages left for midwives went unanswered. They needed to go to extraordinary lengths to find a midwife; some became exasperated and self-referred to the hospital maternity system or returned to the GP.

Public transport was cited as another barrier for women who were new to the region and not confident with navigating an unfamiliar city. Moreover, for some women their cultural beliefs would not allow them to travel alone on public transport and driving was deemed to possibly cause miscarriage. This led to women who did not drive, or did not want to drive, experiencing limited access to LMC midwifery care.

Our participants identified their reality that limited finances influenced ownership of a mobile or landline phone. Limited credit prevented phone calls to multiple LMC midwives to ascertain their availability. This added another layer of tension to accessing a LMC midwife. Limited finance also impacted their ability to afford petrol and parking, especially if needing to drive to a clinic location not on their usual route. The women expressed appreciation at the convenience of being able to access GP care at the same time as seeing the midwife, specifically for their maternal immunisations, mental health and physical ailments. Having this option saved them petrol costs, parking costs and time. Much of the Aotearoa NZ and overseas literature reports that multiparous women do not prioritise accessing early antenatal care for reasons similar to those raised in the current study - time restraints, prioritising the needs of other family/whānau members, and financial limitations (Corbett et al., 2013; Dixon et al., 2013; Haddrill et al., 2014; Hatherall et al., 2016; Ratima \& Crengle, 2013). All the women in our study had pressured, busy lives which impacted their ability to access midwifery care. It could be argued many women have pressured, busy lives; however, the difficulties of the lives of these women were exacerbated by poverty.

This study revealed that the women trusted information, recommendations and referrals from their GP and/or practice nurse. Women wanted reassurance that they would find a "good" midwife. When a midwife was recommended by their GP or practice nurse, the recommendation was perceived to come with an endorsement that the midwife "must be good". The women then accessed the midwife easily, with confidence and with less fear and anxiety about the quality of the care she was to receive.
Through the endorsed recommendation from the GP or practice nurse, the women described a sense of knowing the midwife, despite not having met the midwife previously, and a confidence to contact the midwife to book an appointment when they were ready. However, in order for midwives to be recommended to women, inter-collegial relationships are important. GPs, practice nurses and midwives need to have strong professional relationships to uphold trust and the knowing of each other. "Shared knowing" between health professionals is the basis of a trusted relationship the women then build on when establishing the new LMC midwife relationship (Crowther \& Smythe, 2016). This prior "knowing" helped women feel they could ask the GP or practice nurse important questions about the recommended midwife, which enabled them to build a profile of the midwife and inform their decision-making with regard to choosing a LMC midwife.

The women in this study valued the ease and convenience of having their midwifery care at their GP clinic. Moreover, this came with the advantages of the environment being a familiar place where they knew the staff, as well as lessening the potential for women to be lost between providers. The model of co-location of midwives at GP clinics enabled the women to connect with a LMC midwife who was available and accessible. Co-locating midwives with GP clinics was suggested by Corbett et al. (2013) and Paterson et al. (2012) as a specific health care access strategy for women who live in $\mathrm{CMH}$ high deprivation communities to increase their accessibility to LMC midwifery care. Several studies have identified that health services being provided within a familiar environment is important to people accessing and engaging with the health service (Corbett et al., 2013; McAra-Couper et al., 2018; Priday \& McAra-Couper, 2016; Ratima \& Crengle, 2013; Southwick et al., 2012; Tanuvasa et al., 2013). The women in our study reflected the added advantage of feeling their health history was already known and the GP clinic staff and LMC midwives could share health information. This provided the potential for open dialogue between health professionals, which was valued by the women and enabled their maternity care to remain connected with their primary health care providers. The women witnessed and valued this collegiality, describing that face-to-face communication between LMC midwife and GP clinic personnel added to their sense of completeness of their maternity care, particularly for the hand back process at six weeks post-partum.

Findings from this study confirm that co-located midwifery services at GP clinics were acceptable to, and appreciated by, these participants. Further research could be undertaken to: 1) explore alternative venues for co-located midwifery services that ensure accessibility and acceptability for women living with poverty in Aotearoa NZ; 2) explore the possibility of an accessible internetbased means to assist women seeking midwifery care in a culturally sensitive forum; and 3) assess the role of navigational support workers and early pregnancy midwifery clinics that support navigation to LMC midwives.

\section{STUDY LIMITATIONS}

This study focused on eight multiparous women who live in socioeconomically deprived communities of the Counties Manukau region; hence, our findings might be specific to this geographical region and these women. While concerted efforts were made to recruit women uniquely related to the research question of this study, time restraints may have limited participant numbers and perhaps excluded women who live transiently who were unaware of the study. Finally, this study focused on the experiences of women who utilised the co-location midwifery service and did not explore the experiences of LMC midwives in co-located clinics. 
Although these are limitations, the findings may, regardless, have relevance to other urban localities with high social deprivation.

\section{CONCLUSIONS}

The women in this study found accessing LMC midwifery care early in their pregnancies a daunting journey, made onerous by the effort required to find a LMC midwife. They valued being able to access a clinic which co-located their LMC midwife with their GP, finding this helped them circumvent the maternity care maze. Barriers to accessing early LMC midwifery care included: not knowing how to find a LMC midwife, not having the confidence to contact a midwife, limited finances and limited time. Enablers to accessing early LMC midwifery care included receiving a recommendation from GP clinic personnel, and knowing that the co-location would probably make maternity care convenient, with a smooth transition from GP to LMC midwifery care.

\section{ACKNOWLEDGEMENTS AND CONFLICT OF INTEREST DISCLOSURE}

The authors wish to acknowledge the eight women who took part in this study, and the GP personnel and LMC midwives who assisted in making the study known to potential participants. The authors declare the $2011 \mathrm{MOH}$-commissioned report was funded by the $\mathrm{MOH}$. Otherwise, the authors declare there are no conflicts of interest.

Key points
- Some women encounter
barriers to accessing early LMC
midwifery care, including a
lack of resources and limited
knowledge about finding a
midwife.
A group of women in the
Counties Manukau Health region
expressed the need for help to
access midwifery care.
These women valued midwife
recommendations from their
GP clinic personnel, and having
midwives co-located at their
GP clinic.

\section{REFERENCES}

Aquino, M. R. J. V., Edge, D., \& Smith, D. M. (2015). Pregnancy as an ideal time for intervention to address the complex needs of black and minority ethnic women: Views of British midwives. Midwifery, 31(3), 373-379. https://doi.org/10.1016/j.midw.2014.11.006

Atkinson, J., Salmond, C., \& Crampton, P. (2014). NZDep2013 Index of Deprivation. https://www.health.govt.nz/publication/nzdep2013index-deprivation

Bartholomew, K., Morton, S., Carr, A., Polly, E., Bandara, D. K., \& Grant, C. C. (2015). Provider engagement and choice in the lead maternity carer system: Evidence from Growing Up in New Zealand. Australian and New Zealand Journal of Obstetrics and Gynaecology, 55(4), 323-330. https://doi.org/10.1111/ajo.12319

Berterö, C. (2015). Developing qualitative methods - or "same old wine in a new bottle". International Journal of Qualitative Studies on Health and Well-being, article 27679. http://doi.org/10.3402/qhw.v10.27679
Copland, R. J., Denny, S. J., Robinson, E. M., Crengle, S., Ameratunga, S., \& Dixon, R. (2011). Self-reported pregnancy and access to primary health care among sexually experienced New Zealand high school students. Journal of Adolescent Health, 49(5), 518-524. https://doi. org/10.1016/j.jadohealth.2011.04.002

Corbett, S., Chelimo, C., \& Okesene-Gafa, K. (2013). Barriers to early initiation of antenatal care in a multi-ethnic sample in South Auckland, New Zealand. New Zealand Medical Journal, 127(1404), 53-61. https:// assets-global.website-files.com/5e332a62c703f653182faf47/5e332a62c7 03f67a282fd83c_content\%20(1).pdf

Counties Manukau Health. (2014). Maternity quality and safety programme annual report 2013/2014. https://www.countiesmanukau. health.nz/assets/About-CMH/Reports-and-planning/ Maternity/559fd3bab9/2013-CMH-maternity-quality-safetyprogramme-annual-report.pdf

Crowther, S., \& Smythe, L. (2016). Open, trusting relationships underpin safety in rural maternity a hermeneutic phenomenology study. BMC Pregnancy and Childbirth, 16(1), 307. https://doi.org/10.1186/ s12884-016-1164-9

Dixon, L., Andrews, A., Eddy, A., Guilliland, K., Hendry, C., \& Houston, J. (2013). Changing trends in pregnancy registration for New Zealand women. Journal of Primary Health Care, 6(4), 279-285. https:// doi.org/10.1071/HC14279

Dwyer, S. C., \& Buckle, J. L. (2009). The space between: On being an insider-outsider in qualitative research. International Journal of Qualitative Methods, 8(1), 54-63. https://doi. org/10.1177/160940690900800105

Griffiths, C., McAra-Couper, J., \& Nayar, S. (2013). Staying involved "because the need seems so huge": Midwives working with women living in areas of high deprivation. International Journal of Childbirth, 3(4), 218-232. https://doi.org/10.1891/2156-5287.3.4.218

Guba, E. G., \& Lincoln, Y. S. (1982). Epistemological and methodological bases of naturalistic inquiry. Educational Technology Research and Development, 30(4), 233-252. https://www.jstor.org/ stable/30219846

Haddrill, R., Jones, G., Mitchell, C., \& Anumba, D. (2014). Understanding delayed access to antenatal care: A qualitative interview study. BMC Pregnancy and Childbirth, 14(1), 207. https://doi. org/10.1186/1471-2393-14-207

Hatherall, B., Morris, J., Jamal, F., Sweeney, L., Wiggins, M., Kaur, I., Renton, A., \& Harden, A. (2016). Timing of the initiation of antenatal care: An exploratory qualitative study of women and service providers in East London. Midwifery, 36, 1-7. https://doi.org/10.1016/j. midw.2016.02.017

Jackson, C. (2011). Antenatal care in Counties Manukau DHB: A focus on primary antenatal care. Counties Manukau District Health Board. http:// www.countiesmanukau.health.nz/assets/About-CMH/Performance-andplanning/health-status/2011-antenatal-care-CMDHB.pdf

Lindquist, A., Kurinczuk, J., Redshaw, M., \& Knight, M. (2014). Experiences, utilisation and outcomes of maternity care in England among women from different socio-economic groups: Findings from the 2010 National Maternity Survey. BJOG: An International Journal of Obstetrics and Gynaecology, 122(12), 1610-1617. https://doi. org/10.1111/1471-0528.13059

Lovell, A. (1996). Power and choice in birthgiving: Some thoughts. British Journal of Midwifery, 4(5), 268-272. https://doi.org/10.12968/ bjom.1996.4.5.268

Makowharemahihi, C., Lawton, B. A., Cram, F., Ngata, T., Brown, S., \& Robson, B. (2014). Initiation of maternity care for young Maori women under 20 years of age. New Zealand Medical Journal, 127(1393), 52-61. http://journal.nzma.org.nz/journal/127-1393/6107/

McAra-Couper, J., Farry, A., Marsters, N., Otukolo, D., Clemons, J., \& Smythe, L. (2018). Pasifika women's choice of birthplace. New Zealand College of Midwives Journal, 54, 15-21. https://doi.org/10.12784/ nzcomjnl54.2018.2.15-21

Midwifery Council of New Zealand. (2010). The Midwifery Scope of Practice. https://midwiferycouncil.health.nz/Public/01.-Midwiferyin-Aotearoa-New-Zealand/The-Midwifery-Scope-of-Practice. aspx?WebsiteKey=f0308050-1256-4559-908a-2c0b3a7fd7e5 Ministry of Health. (2017). The 5th annual report for the National Maternity Monitoring Group. https://www.health.govt.nz/system/files/ documents/publications/nmmg-annual-report-2017-jun18_-_copy.pdf Ministry of Health. (2018). National Maternity Monitoring Group. https://www.health.govt.nz/our-work/life-stages/maternity-services/ national-maternity-monitoring-group 
Ministry of Health. (2020). Report on Maternity web tool. https:// minhealthnz.shinyapps.io/Maternity_report_webtool/

National Institute for Health and Clinical Excellence. (2010). Pregnancy and complex social factors: A model for service provision for pregnant women with complex social factors. https://www.nice.org.uk/guidance/cg110

Paterson, R., Candy, A., Lilo, S., McCowan, L., Naden, R., \& O'Brien, M. (2012). External review of maternity care in the Counties Manukau District. Counties Manukau District Health Board. http://www. countiesmanukau.health.nz/assets/About-CMH/Reports-and-planning/ Maternity/2012-CMH-external-report-maternity-care-review.pdf Perinatal and Maternal Mortality Review Committee. (2018). Twelfth annual report of the Perinatal and Maternal Mortality Review Committee. https://www.hqsc.govt.nz/assets/PMMRC/Publications/12th-PMMRCreport-final.pdf

Priday, A., \& McAra-Couper, J. (2016). A successful midwifery model for a high deprivation community in New Zealand: A mixed methods study. International Journal of Childbirth, 6(2), 78-92. http://doi. org/10.1891/2156-5287.6.2.78

Ratima, M., \& Crengle, S. (2013). Antenatal, labour, and delivery care for Māori: Experiences, location within a lifecourse approach, and knowledge gaps. Primatisiwin: A Journal of Aboriginal and Indigenous Community Health, 10(3), 353-366. https://journalindigenouswellbeing. com/wp-content/uploads/2013/02/08RatimaCrengle.pdf

Smythe, L. (2012). Discerning which qualitative approach fits best. New Zealand College of Midwives Journal, 46, 5-12. https://www.midwife.org. nz/wp-content/uploads/2019/01/JNL-46-June-2012.pdf

Southwick, M., Kenealy, T., \& Ryan, D. (2012). Primary care for Pacific people: A Pacific and health systems approach. Pacific Perspectives. https:// www.health.govt.nz/publication/primary-care-pacific-people-pacific-andhealth-systems-approach

Tanuvasa, A. F., Cumming, J., Churchward, M., Neale, J., \& Tavila, A. (2013). Samoan women's attitudes towards antenatal and midwifery care. British Journal of Midwifery, 21(10), 710-721. https://doi. org/10.12968/bjom.2013.21.10.710

Temple, B. (1997). 'Collegial accountability' and bias: The solution or the problem? Sociological Research, 2(4), 8-14. https://doi.org/10.5153/ sro. 144

Thorne, S. (2008). Interpretive description. Left Coast Press.

Thorne, S., Con, A., McGuinness, L., McPherson, G., \& Harris, S. R. (2004). Health care communication issues in multiple sclerosis: An interpretive description. Qualitative Health Research, 14(1), 5-22. https:// doi.org/10.1177/1049732303259618

Thorne, S., Kirkham, S. R., \& O'Flynn-Magee, K. (2004). The analytic challenge in interpretive description. International Journal of Qualitative Methods, 3(1), 1-11. https://doi.org/10.1177/160940690400300101

\section{Accepted for Publication May 2021}

Priday, A., Payne, D., \& Hunter, M. (2021). A daunting journey: A qualitative comparative study of women's experiences of accessing midwifery care. New Zealand College of Midwives Journal, 57, 27-33 https://doi.org/10.12784/nzcominl57.2021.4.27-33 